\title{
Reinhard Bouché:
}

\section{Die Fernleihe geht neue Wege}

\section{Ein Anfang wird gemacht}

Seit dem 6. Mai 2002 findet in der Württembergischen Landesbibliothek ein kontinuierlicher Umbruch in den Organisationsabläufen der Fernleihe statt. Es begann mit der Einführung des elektronischen Bestellformulars, was folgende unmittelbare Konsequenzen hatte:

$\Rightarrow$ keine Ausgabe von Fernleihscheinen mehr an der Auskunft.

$\Rightarrow$ Ausdruck der FL-Scheine im traditionellen 'outfit' über einen Netzdrucker.

$\Rightarrow$ Verbuchung der FL-Gebühren auf den Gebührenkonten der Benutzer im Ausleihsystem.
$\Rightarrow$ Einsicht des Benutzers in seine vollständigen Bestellangaben über Links im Online-Kontoauszug.

$\Rightarrow$ Sukzessiver Abbau der noch verbliebenen konventionellen Karteien in der Fernleihe.

Nicht lange nach dieser Umstellung wurde auf Initiative der Stuttgarter Bibliotheksdirektoren das Projekt 'Zentrale Verbund-FernLeihe' (ZFL) vom Bibliotheksservicezentrum (BSZ) in Angriff genommen. Entsprechend dem Vorbild von Nordrhein-Westfalen und Bayern sollte ein im BSZ betriebener Server die Fernleihen entgegennehmen und elektronisch auf den im Verbundsystem ermittelten Leitweg schicken.

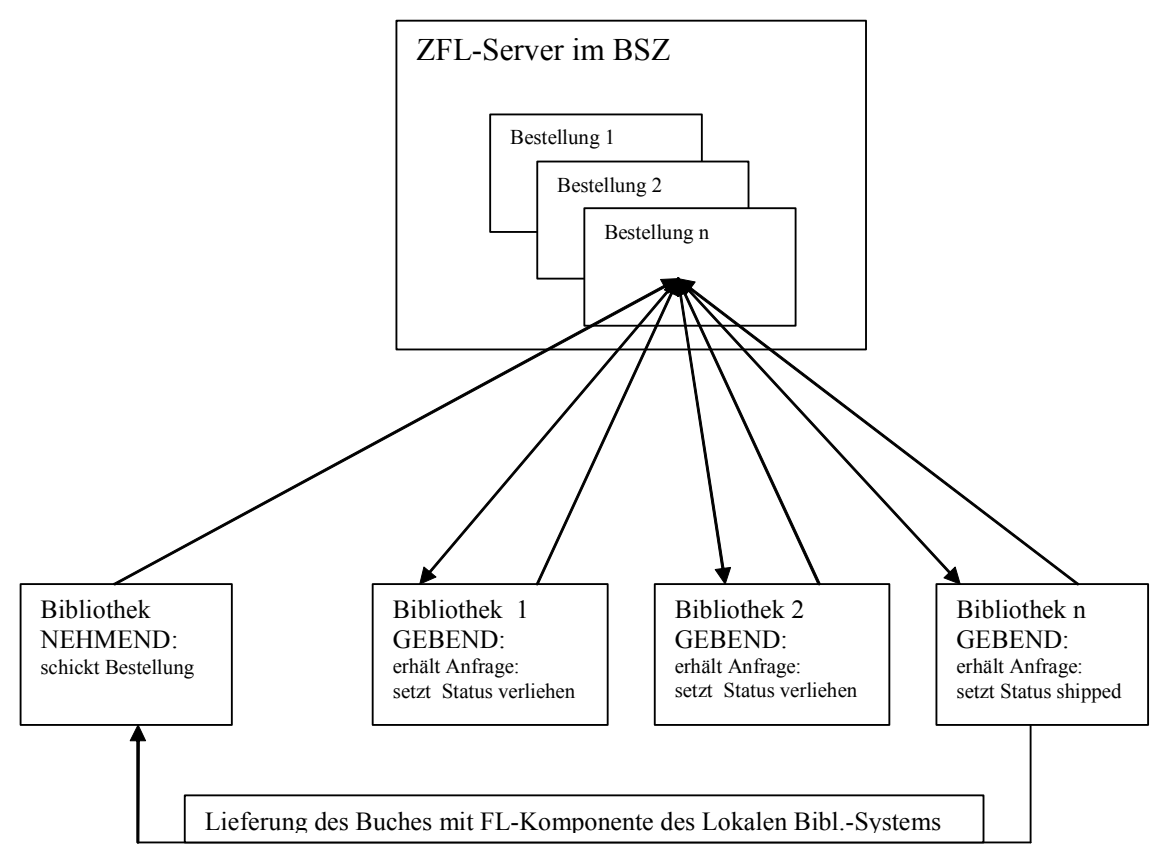


Die eigens für dieses Projekt eingerichtete Arbeitsgruppe machte sich am 23. September 2002 mit der 1. Sitzung in der WLB an die Arbeit und am 17. März 2003 konnte die erste gebende ZFLFernleihe in der WLB in folgenden Schritten erledigt werden:

1. Aufruf des entsprechenden Titels im OPAC der WLB und Überprüfung der Signatur(en) im Ausleihsystem auf Verfügbarkeit.

2. Falls ausleihbares Exemplar vorhanden: Ausdruck eines FL-Scheins in der Fernleihe als Bestellunterlage für das Magazin. Danach Verbuchung in Bibdia und normale Verschickung.

3. Falls nicht: elektronische Weiterleitung der Bestellung über das ZFLSystem.

\section{Automatisierung der geben- den Fernleihe}

Die Erledigung der ersten ZFL-Fernleihe dieser Art war natürlich spannend, allerdings war der Ausdruck der FLScheine als PDF-Dokument bei steigender Zahl sehr zeitraubend und ein zusätzlicher Aufwand im Vergleich zur konventionellen Fernleihe.

Aus diesem Dilemma befreite uns eine Programmentwicklung aus der UB Freiburg, die genau die Schritte 1-3 automatisierte:

Durch Überprüfung der Bestellung am OLIX/OPAC konnten auch Mehrfachex- emplare berücksichtigt werden. Falls kein ausleihbares Exemplar vorhanden war, wurde die Bestellung automatisch weitergeschickt; im anderen Fall wurde vom Programm der Ausdruck eines FLScheins angestoßen.

Dieses Programm wurde in der WLB angepasst und am 1. August 2003 im Betrieb genommen. Es 'erledigt' zum jetzigen Zeitpunkt ca. 120 gebende Fernleihen pro Tag, Tendenz steigend.

Alle ZFL-Bestellungen auf ausleihbare Monographien können seitdem direkt im Magazin auf einem gesonderten Drucker im 1.UG/Abschnitt 5 ausgedruckt werden.

Die anderen Bestellungen, die noch einer manuellen Nachbehandlung bedürfen (z.B. die Ermittlung der genauen Bandangaben bei mehrbändigen Werken oder Zeitschriften) werden in der Fernleihe ausgedruckt.

Ab dem 12. Juli 2004 wurde dann ein weiterer Schritt über den SWB-Verbund hinaus getan: der Bayerische Bibliotheksverbund (BVB) übergibt seit diesem Tag als ,Nehmende Bibliothek' Fernleihen aus seinem Verbund an das ZFL-System des SWB. Mittlerweile bearbeitet die WLB täglich ca. 15 Fernleihen aus dem BVB.

Abbildung 1 zeigt einen Auszug aus dem Protokoll des einmal pro Tag stattfindenden Programmlaufs für die gebende ZFL-Fernleihe: 


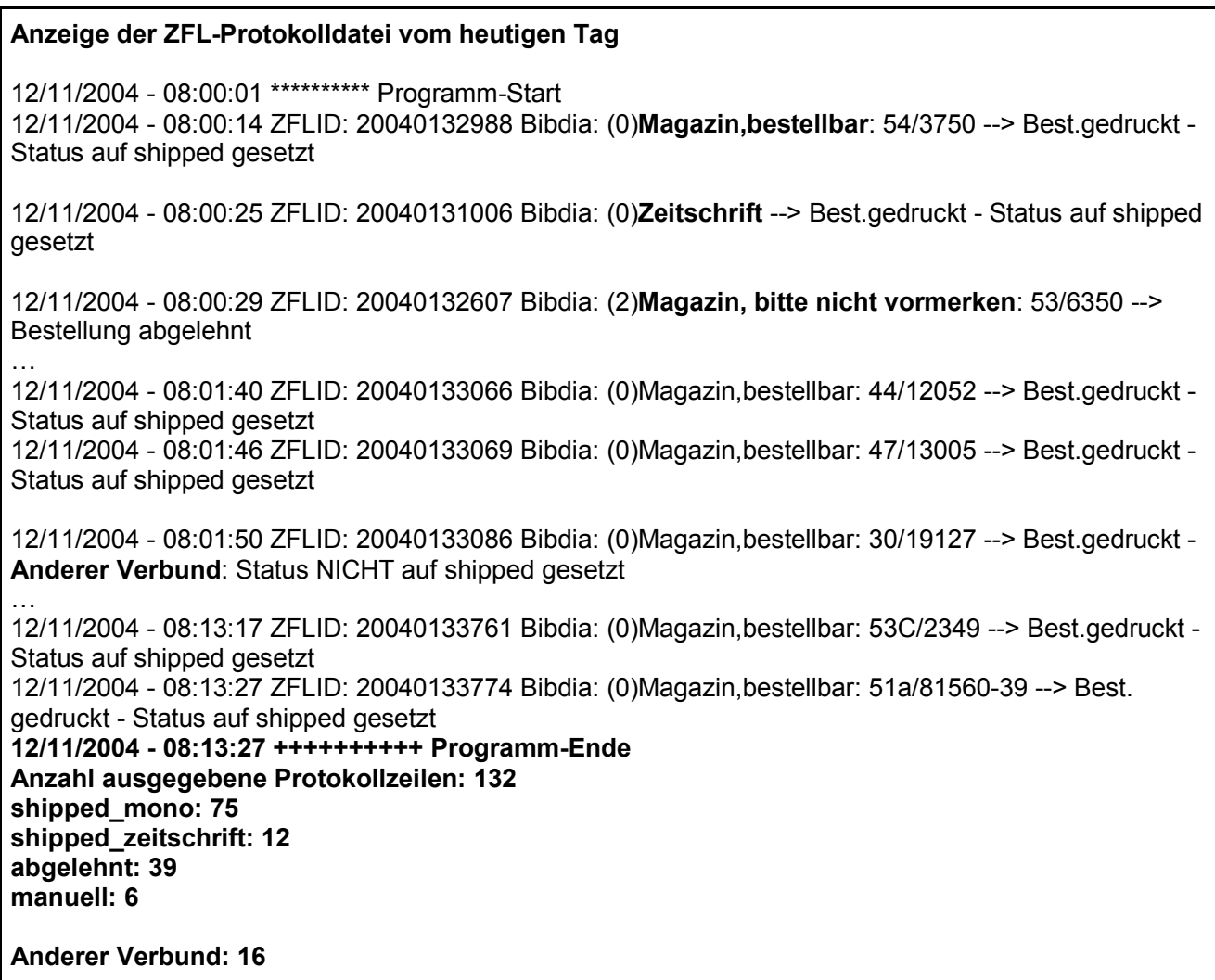

Abbildung 1

\section{Beschleunigung der nehmen- den Fernleihe}

Auch nach Einführung der OnlineFernleihbestellung in der WLB am 6.5.2002 musste zunächst noch jede Fernleih-Bestellung unserer Benutzer in der Fernleihe manuell in Bibdia eingetragen werden, und zwar einmal die Bestellung selbst und dann noch die Gebühr. Auch hier hat uns wieder die UB Freiburg weitergeholfen mit einem Programm, dass diese Einträge in Bibdia automatisch vornimmt: seit dem 6 . Oktober 2003 kann eine Fernleihe direkt nach dem Bibliographieren entsprechend dem festgelegten Leitweg verschickt werden und die Benutzer können spätestens 3 Minuten nach $\mathrm{Ab}$ schicken der FL-Bestellung diese Fern- leihe in ihrem Bestellkonto von Bibdia wiederfinden und anhand der bibliographischen Daten identifizieren.

Wichtigstes Desiderat für die nehmende Fernleihe war jedoch das Ziel, den Benutzer selbst eine Bestellung ausführen zu lassen, die programmgesteuert in das ZFL-System überführt werden konnte.

Eine Voraussetzung dafür ist, dass der Benutzer selbst entweder im StOPAC oder im WebPAC des Verbunds den Titel recherchiert, den er per Fernleihe bestellen will.

Als Ausgangspunkt hierfür bietet sich zunächst die BISSCAT-Recherche im StOPAC an, da der Benutzer nach einer ergebnislosen BISSCAT-Recherche im WLB-OPAC routinemäßig auf 
die Recherche im StOPAC verwiesen wird. An zwei Stellen des StOPAC bieten wir deshalb für unsere Benutzer Links für eine Fernleihbestellung an.

\section{Kein Treffer im StOPAC}

Bei null Treffern im StOPAC gibt es einen Link in den WebPAC des SWB speziell für die Fernleihbestellung eines Buchs, Zeitschriften sind vorerst noch ausgenommen (Abbildung 2).

Katalogauswahl: StOPAC (Stuttgarter Online-Katalog)

Suchanfrage: find ti=gletscherspaltung

Trefferanzahl 0

Thre Suchanfrage führte zu 0 Treffern.

Bitte prüfen Sie Thre Eingaben und starten einen neuen Versuch.

Weitersuche im Südwestdeutschen Bibliotheksverbund

Fernleihbestellung (Info):

fur Nutzer der Württembergischen Landesbibliothek

$\Rightarrow$ Fernleihbestellung mit Verbundrecherche (Bucher)

- Bestellformular Fernleihe (Zeitschriften, Aufsatze)

furr Nutzer der Uni-Bibliothek Stuttgart

- Fernleihbestellung mit Verbundrecherche

- Bestellformular Fernleihe

fur Nutzer der Uni-Bibliothek Hohenheim

- Fernleihbestellung mit Verbundrecherche (Bücher)

- Bestellformular Fernleihe (Zeitschriften, Aufsatze)

Abbildung 2

Dieser Link führt auf das leere Suchformular des WebPAC und übergibt als Aufrufparameter eine Kennung für die WLB. Der Benutzer recherchiert nun im WebPAC. Eine Volltitelanzeige für Monographien enthält jetzt neben den üblichen Bestandsangaben einen Fernleihlink (Abbildung 3).

Dies ist - obwohl aus der Bildschirmanzeige nicht ersichtlich - ein spezieller WLB-Fernleihlink, der auf ein FL-
Bestellformular der WLB mit vorausgefüllten bibliographischen Angaben zeigt (Abbildung 4).

Eine von diesem Formular erzeugte Bestellung kann dann von einem lokalen Programm in das ZFL-System eingegeben werden, weil sie bereits mit der SWB-Identnr. versehen ist. 
Bouché: Die Fernleihe geht neue Wege

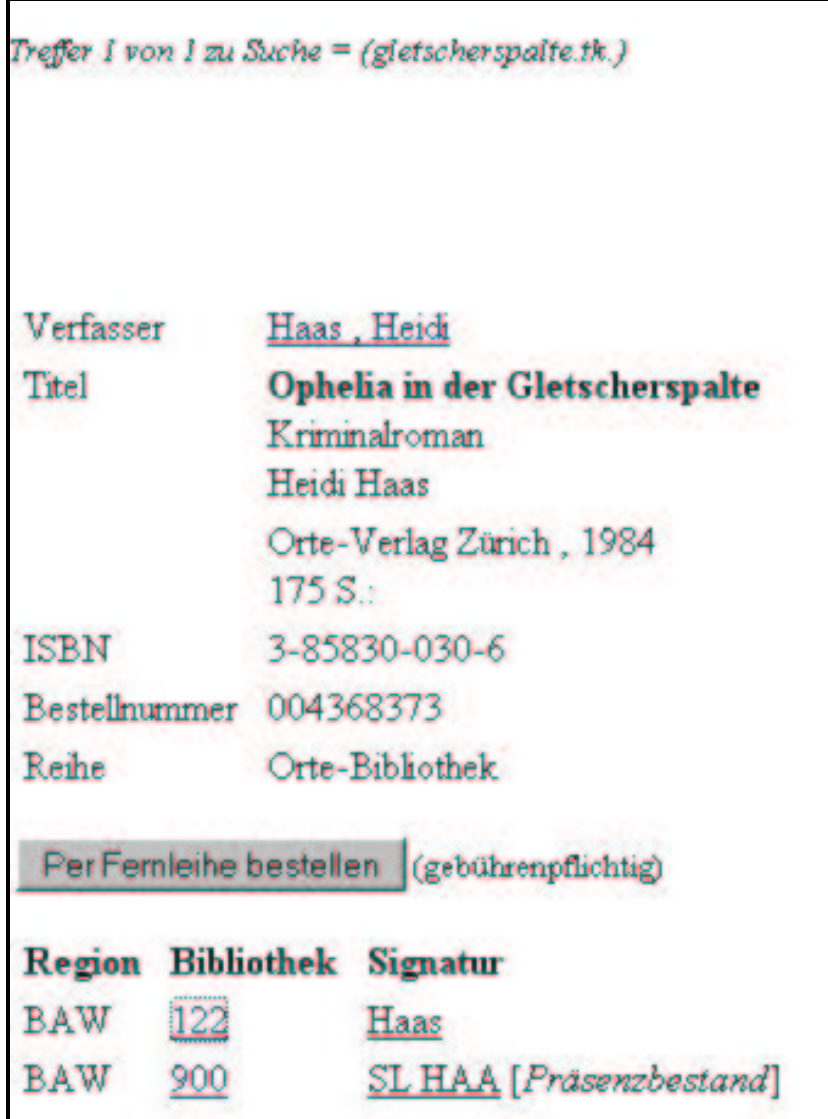

Abbildung 3

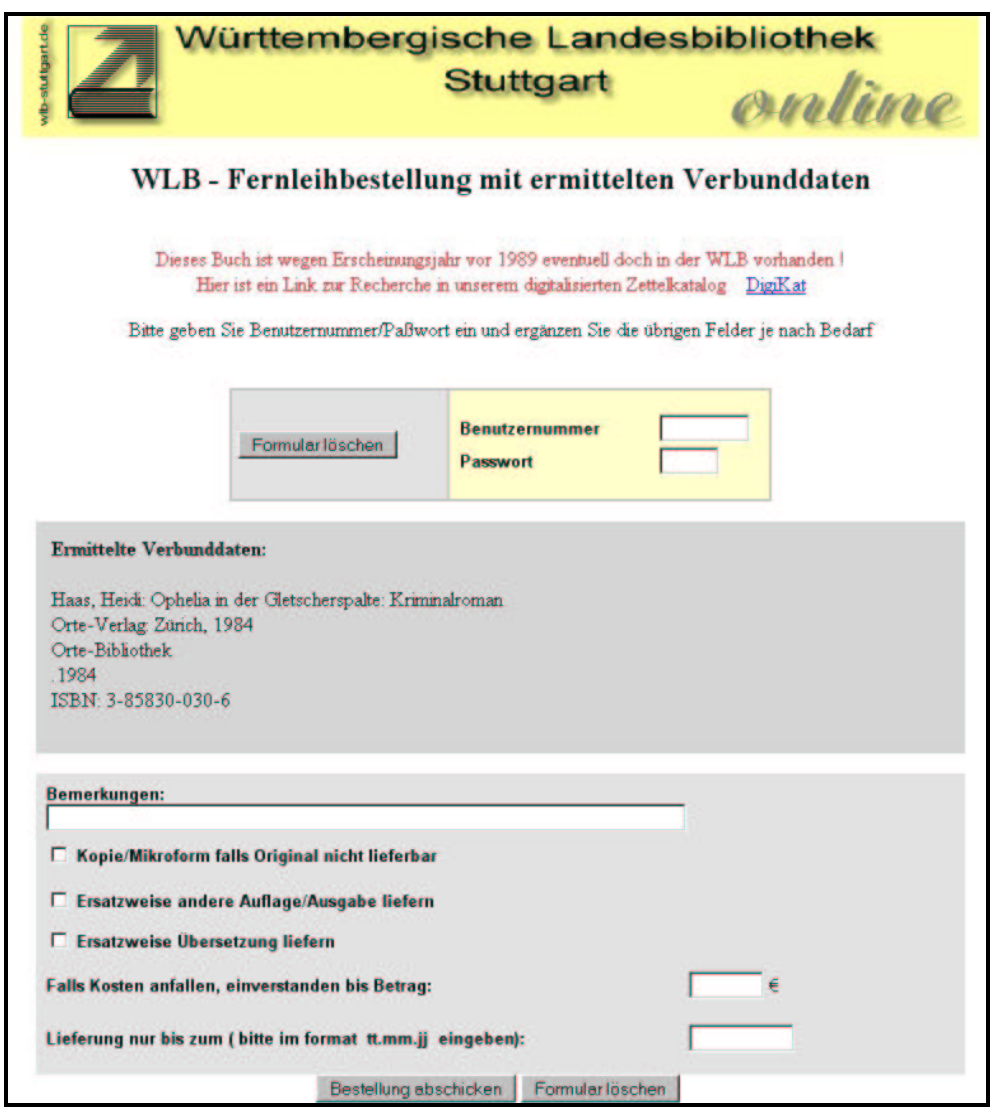

Abbildung 4 


\section{Treffer im StOPAC}

Bei einem Treffer im StOPAC, der keinen Bestand für die WLB aufweist, wird für die Benutzer der WLB der Link 'Diesen Titel per Fernleihe bestellen' angeboten. Hier kann ohne Umweg über den WebPAC direkt das WLBFormular mit den vorausgefüllten bibliographischen Daten angesteuert werden. Für die Benutzer der UB Stuttgart und der UB Hohenheim gibt es die entsprechenden Links.

Falls das Erscheinungsjahr vor 1980 liegt, wird bei der WLB noch zusätzlich der Link zum DigiKat angeboten. Über die Info-Links wird jeweils ausführlich auf die Bedingungen der Fernleihe hingewiesen.
Aber auch Fernleihbestellungen, die weder im StOPAC noch im WebPAC recherchiert wurden, können automatisiert an das ZFL-System weitergeleitet werden, wenn sie im Fall von Monographien eine ISBN-Nummer enthalten. Mit dieser ISBN-Nummer kann mit lokalem Programm im WebPAC recherchiert und bei einem eindeutigen Treffer und ausleihbarem Bestand unmittelbar eine ZFL-Bestellung generiert werden.

Diese skizzierten Möglichkeiten einer Fernleihe werden seit Mitte April 2004 angeboten und auch gut genutzt, fast möchte man sagen 'zu gut genutzt', denn leider gibt es auch negative Effekte:

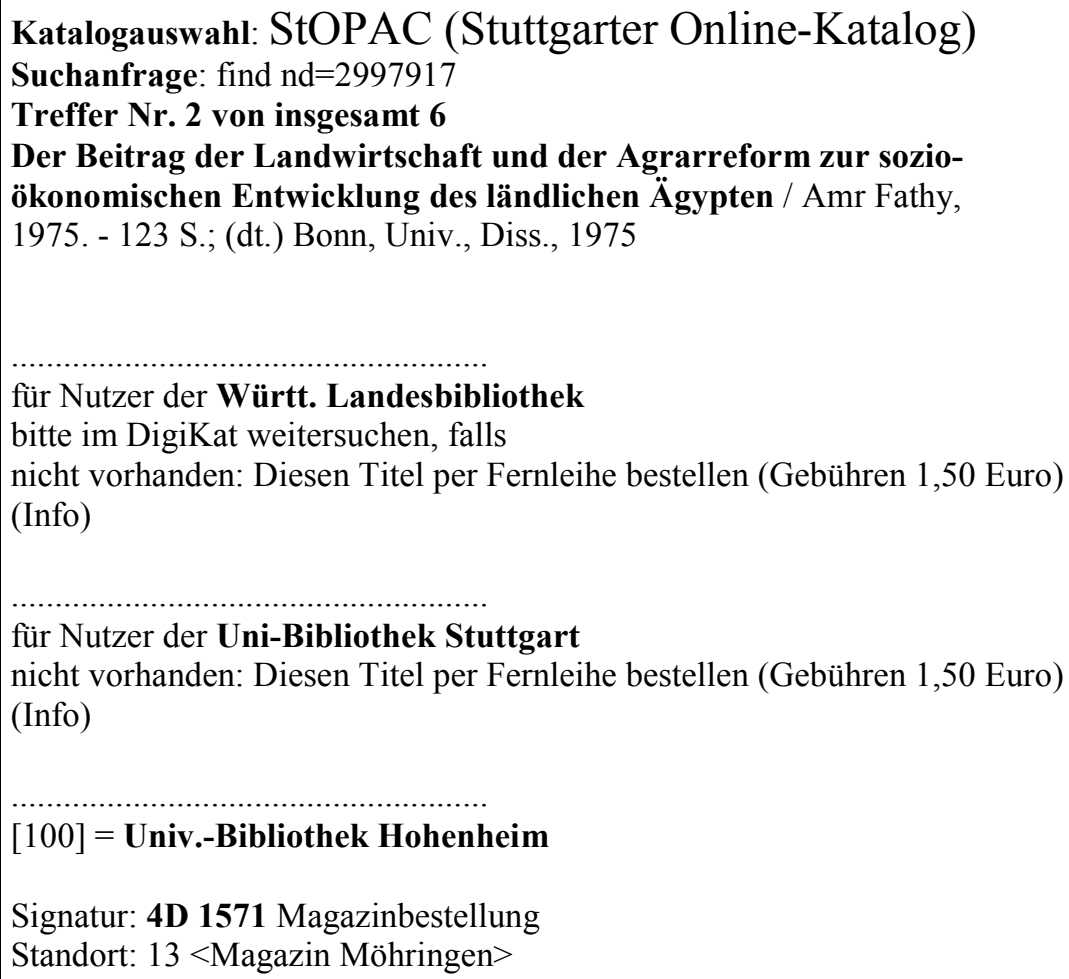


Die Benutzer bestellen über den WebPAC oder auch den StOPAC Bücher, die in der WLB vorhanden sind. Entweder sie interpretieren die Bestandsangaben im WebPAC nicht richtig oder aber sie recherchieren bei Erscheinungsjahren vor 1989 nicht im DigiKat, obwohl sie bei den Bestellformularen ausdrücklich darauf hingewiesen werden.

Eine intensive Aufklärung der Benutzer über den Aufwand einer Fernleihe, der ja bei weitem nicht durch $1,50 €$ abgedeckt wird, tut hier not.

Eine weitere, unschöne Lücke im flotten ZFL-System ist die Tatsache, dass noch nicht alle Fernleihbibliotheken der Südwest-Region als gebende Bibliothek im ZFL-System teilnehmen, obwohl der Einstieg in das ZFL-System

\section{FL-Statistik der WLB gebend und nehmend für Januar - September 2004}

- gebend

\begin{tabular}{|l|l|l|}
\hline & Gesamt & Positiv erledigt \\
\hline ZFL + Konventionell & 32.212 & 17.546 \\
\hline ZFL & 14.877 & 9.015 \\
\hline
\end{tabular}

- nehmend

\begin{tabular}{|l|l|l|}
\hline & Gesamt & Positiv erledigt \\
\hline ZFL + Konventionell & 16.546 & 13.351 \\
\hline ZFL & 4.204 & 3.783 \\
\hline
\end{tabular}

als gebende Bibliothek lediglich einen Internetanschluss, den Acrobat Reader Version 5 sowie einen Laserdrucker erfordert.

Deshalb ist momentan der ZFL-Status , nicht lieferbar' leider nicht identisch mit der Aussage, dass das Buch in der Südwest-Region nicht fernleihbar vorhanden ist. Eine - hoffentlich bald verschwindende - verwirrende Situation für die Fernleihabteilungen.

Bei der gebenden ZFL-Fernleihe steht die WLB in der Region des SWBVerbunds an erster Stelle.

Ein deutlicher Hinweis auf den guten Bestand der WLB und ihre Bedeutung für die ganze Region. 


\section{Ausblick}

So wie der BVB jetzt schon FLBestellungen an unser ZFL-System automatisiert weitergibt, sollen in absehbarer Zeit auch unsere ZFL-Bestellungen automatisiert an das ZFLSystem des BVB weitergegeben werden. Im Augenblick muss man dazu noch manuell eingreifen:

eine ZFL-Fernleihe, die den Leitweg vergeblich durchlaufen und den Status, nicht lieferbar' erhalten hat, kann per Knopfdruck im BVB recherchiert und bei positivem Ergebnis an das ZFLSystem des BVB weitergeleitet werden.
Die ZFL-Fernleihen von Zeitschriftenaufsätzen sind auch SWB-intern noch problematisch, da der Abgleich zwischen den Bestelldaten und den Bestandsangaben der jeweiligen Bibliothek noch sehr unzureichend ist, d.h. das ZFL-System produziert fehlerhafte Leitwege. Natürlich kann eine 'falsche' Aufsatzbestellung von uns elektronisch weitergeschickt werden - sie muss aber vorher am Bestand mit entsprechendem Aufwand recherchiert werden. Die Lösung liegt hier in einer Z39.50-Schnittstelle des ZFL-Systems mit der ZDB, die eine bessere Überprüfung möglich machen soll.

Sibylle Baur:

\section{Die Fernleihe geht neue Wege: Bericht aus der Praxis}

\section{Bestellungen unserer eigenen Benutzer:}

Mit der Einführung des Elektronischen Bestellformulars stürzten sich unsere Benutzer auf diese Möglichkeit der Bestellung, die Anzahl der Bestellungen stieg sprunghaft an.

\section{Fernleihe, was ist das eigent- lich?}

Fernleihe bedeutet, wir besorgen für unsere Benutzer Bücher ,die wir nicht im Bestand haben, aus anderen Bibliotheken und legen sie in der Landesbibliothek zur Abholung bereit.
Für viele unserer Benutzer aber war mit Einführung des Onlinebestellformulares klar, Fernleihe bedeutet: ich bestelle von zu Hause aus ein Buch aus dem Bestand der Württembergischen Landesbibliothek und bekomme das Buch dann dorthin geliefert. Da gab es einiges an Aufklärungsbedarf !

\section{Online-Fernleihe über den ZFL-Server des BSZ}

Nach der Inbetriebnahme der Onlinefernleihe über den ZFL-Server des Bibliotheksservicezentrums Baden-Württemberg konnten die Bestellungen auf 